\title{
Evolution de la capacité de germination des semences de mauvaises herbes enfouies dans le sol
}

\author{
Jean-Pierre LONCHAMP, René CHADOEUF \& Gilbert BARRALIS
}

avec la participation technique de Maurice BourLIER

I.N.R.A., Laboratoire de Malherbologie, B.V. 1540, F 21034 Dijon Cedex

Des semences de 4 espèces de mauvaises herbes, Aethusa cynapium L., Alopecurus myosuroides Huds., Euphorbia exigua L. et Matricaria perforata Mérat, sont disposées dans des sachets de tergal enfouis dans le sol à une profondeur de 10 et $25 \mathrm{~cm}$. Toutes les 3 semaines, et durant 28 mois, des sachets sont prélevés pour évaluer en laboratoire la capacité germinative des semences restantes. Parallèlement, la chronologie de levée de ces mêmes espèces est étudiée sur 2 dispositifs : dans un peuplement naturel sous culture assolée et dans un peuplement artificiel créé par enrichissement en semences, sous monocultures de blé d'hiver et d'orge de printemps. L'observation des levées est effectuée régulièrement sur ces parcelles.

Les semences de matricaire disparaissent (probablement par germination in situ) à un taux annuel de 17 p. 100 : les semences de toutes les autres especes se conservent.

Hormis les semences de matricaire placées à la lumière qui germent à 100 p. 100, la capacité germinative évolue dans le temps:

1) de façon continue chez la matricaire (obscurité) et la petite ciguë (lumière) dans le sens d'une augmentation avec la durée d'enfouissement;

2) de façon cyclique à périodicité annuelle chez la petite ciguë (obsc.), l'euphorbe et le vulpin. La capacité germinative est corrélée à la température, à l'humidité du sol ainsi qu'à la durée d'enfouissement ; des droites de régressions multiples ont été calculées.

L'étude chronologique des levées au champ montre que :

1) les levées correspondent aux époques de la capacité germinative maximale ; l'étalement dans le temps est limité par la disparition, par germination, des semences placées dans des conditions de germination favorables ;

2) en première approximation, l'âge de l'infestation intervient assez peu sur la chronologie des levées.

Mots clés additionnels : Enfouissement, chronologie des levées, Aethusa cynapium L., Alopecurus myosuroides Huds., Euphorbia exigua L., Matricaria perforata Mérat.

\section{Germinability of buried weed seeds}

Seeds of 4 weeds, Aethusa cynapium L., Alopecurus myosuroides Huds., Euphorbia exigua L. and Matricaria perforata Mérat were buried in the soil at 10 and $25 \mathrm{~cm}$ depth. At 3-week intervals and over a 28-month period, seeds were extracted from the soil for examination of their germinability. Concomitant investigations were made of seedling emergence in the field in 2 experiments : a naturally occurring weed population with a crop rotation and an artificially established population with a continued winter wheat crop and a continued spring barley crop. On these plots, the number of seedlings which emerged was regularly observed.

For Matricaria, the buried seeds disappeared (probably by germination) with an annual rate of $17 \%$; the buried seeds of the 3 other weeds were preserved.

Apart from the seeds of Matricaria which showed $100 \%$ germination when incubated in the light, the germinability of seeds recovered from the soil changed :

1) seeds of Matricaria incubated in the dark and seeds of Aethusa incubated in light showed an increase in the rate of germination with increasing burial time ;

2) seeds of Aethusa incubated in the dark and seeds of Euphorbia and Alopecurus incubated in the light or in the dark showed a cyclic annual pattern. The germination capacity correlated with soil temperature and soil moisture during burial and also with burial time. To find the principal factors affecting germination a regression analysis was used. The field emergence pattern showed that :

1) seedling emergence occurred when the germination ability was maximum. The emergence period was limited by the germination of the seeds which were naturally put in favourable conditions for germination ; 2) the burial time did not seem to modify the emergence pattern.

Additional key words : Buried seeds, emergence pattern, Aethusa cynapium L., Alopecurus myosuroides Huds., Euphorbia exigua L., Matricaria perforata Mérat. 


\section{INTRODUCTION}

La différence de comportement germinatif de semences sèches ou conservées dans le sol a été clairement mise en évidence par les travaux de WESSON \& WAREING (1969). La capacité germinative des semences enfouies évolue lorsque la durée de conservation augmente (TAYLORSON, 1970, 1972; STOLLER \& WAX, 1974) et on observe fréquemment que ces fluctuations revêtent un caractère cyclique de périodicité annuelle (COURTNEY, 1968; POPAY \& ROBERTS, 1970 ; BASKIN \& BASKIN, 1979, 1981 $a, b$ ). Ces fluctuations de la capacité germinative sont interprétées par les différents auteurs comme l'apparition et l'élimination de dormances induites par le milieu de conservation. Dans le sol, les principaux facteurs invoqués pour leur action directe ou indirecte sur les semences sont : la température et l'humidité (KARSSEN, 1982).

De précédents travaux menés au laboratoire sur une quinzaine d'espèces fréquentes dans les cultures annuelles (CHADOEUF et al., 1980 ; LONCHAMP \& GORA, 1980b), ont montré que le caractère saisonnier de l'apparition de la dormance était lié à la température et/ou à l'humidité du sol.

Ces expériences ne nous permettaient pas d'estimer la durée pendant laquelle les semences devaient être soumises à un état de l'un ou l'autre de ces facteurs pour acquérir ou dissiper cet état dormant.

Afin de préciser, dans les conditions naturelles, la rapidité avec laquelle les semences réagissent à une brusque variation de la température ou de l'humidité du sol, des semences ont été enfouies en plein champ et leur capacité de germination a été estimée périodiquement et corrélée à la température et à l'humidité du sol. Parallèlement, les levées au champ de ces mauvaises herbes ont été observées sur 2 parcelles, afin de comparer la capacité germinative et l'apparition de plantules.

\section{MATÉRIELS ET MÉTHODES}

\section{A. Méthode d'estimation de la capacité germinative}

En 1980, on a récolté à maturité des semences d'Alopecurus myosuroides Huds. le 8/07, de Matricaria perforata Mérat le 8/07, d'Euphorbia exigua L. le $20 / 08$ et $d$ 'Aethusa cynapium L. le 16/09, dans des champs envahis par ces mauvaises herbes. Ces semences sont conservées au sec à la température du laboratoire jusqu'à la date de leur utilisation, le 20/10/80. Cinq cents semences de chaque espèce sont alors placées séparément dans des sachets de voile de tergal de $5 \times 7 \mathrm{~cm}$, contenant environ $35 \mathrm{~g}$ de terre tamisée à $2 \mathrm{~mm}$; au total 204 sachets par espèce sont ainsi préparés. Ils sont enfouis dans des corbeilles ajourées pour transplantation d'arbustes à 2 niveaux $(-10$ et - $25 \mathrm{~cm}$ par rapport à la surface de la corbeille) et à raison de 2 espèces par corbeille. Les corbeilles sont ensuite placées en terre en plein champ, le bord supérieur de chacune affleurant le niveau du sol.

Toutes les 3 semaines environ à partir de la date d'enfouissement, et durant 28 mois, des corbeilles sont retirées du sol à raison de 3 répétitions par espèce. Les sachets de semences sont extraits en chambre noire munie d'un éclairage vert inactinique. Après léger brassage du contenu des sachets dans l'eau, les semences sont séparées de la terre et prélevées en vue de tests germinatifs et de l'estimation des pertes durant leur conservation.

Les tests de germination sont effectués sur papier buvard humide (Germaflor, Muller et Fils), en bacs de germination de type Jacobsen, à raison de 100 semences par répétition. Les bacs sont placés en enceintes climatisées à alternance de température : $15-10^{\circ} \mathrm{C}$ (12 h-12 h), soit à l'obscurité totale soit en présence de lumière durant la phase thermique la plus élevée de la thermopériode. La source lumineuse est constituée de tubes fluorescents (type Blanc Industrie, Philips) et de lampes à incandescence, le tout dispersant une énergie de $34 \mathrm{~W} . \mathrm{cm}^{-2}$.

La durée des tests est de $28 \mathrm{j}$; les comptages des semences germées sont effectués tous les 2 ou $3 \mathrm{j}$. On traduit le résultat des comptages en taux de germination de semences morphologiquement normales; ils traduisent la capacité germinative dans les conditions précisées.

\section{B. Méthode d'estimation des levées au champ}

La chronologie des levées, appréciées par l'analyse quantitative de la flore de surface, est réalisée dans 2 dispositifs expérimentaux :

- le $1^{\text {er }}$, à partir du peuplement naturel d'une parcelle régulièrement assolée (pois de printemps, blé d'hiver, orge de printemps) ;

- le $2^{\mathrm{e}}$, à partir d'un peuplement artificiel obtenu par semis de 3500 à 4500 semences $/ \mathrm{m}^{2}$ selon les espèces. Les graines ou caryopses ont été récoltés durant l'été 1979 et incorporés par un labour de $30 \mathrm{~cm}$ en novembre 1979. Les levées ont été observées sur 2 parcelles conduites chacune en monocultures de blé d'hiver et d'orge de printemps.

Les comptages de levées de plantules sont effectués toutes les 3 semaines, dans 34 rectangles permanents $(50 \times 12 \mathrm{~cm})$ tout au long de l'année dans le cas du peuplement artificiel, mais seulement durant le cycle végétatif des cultures dans le cas du peuplement naturel.

\section{Mesure de la température et de l'humidité du sol}

\section{Température}

Les relevés climatiques de l'aire météorologique du domaine expérimental ont été utilisés, particulièrement les mini et maxi à $-10 \mathrm{~cm}$ (fig. 1), leur médiane $(\operatorname{mini}+\operatorname{maxi}) / 2$, ainsi que la moyenne journalière sous abri. Ces dernières valeurs nous ont permis d'établir des moyennes sur les $10,20,30,40$ et $60 \mathrm{j}$ qui ont précédé l'extraction des semences. Ces moyennes nous permettent de calculer les coefficients de corrélation entre la capacité germinative des semences lors d'une extraction et la température du sol durant ces périodes. 


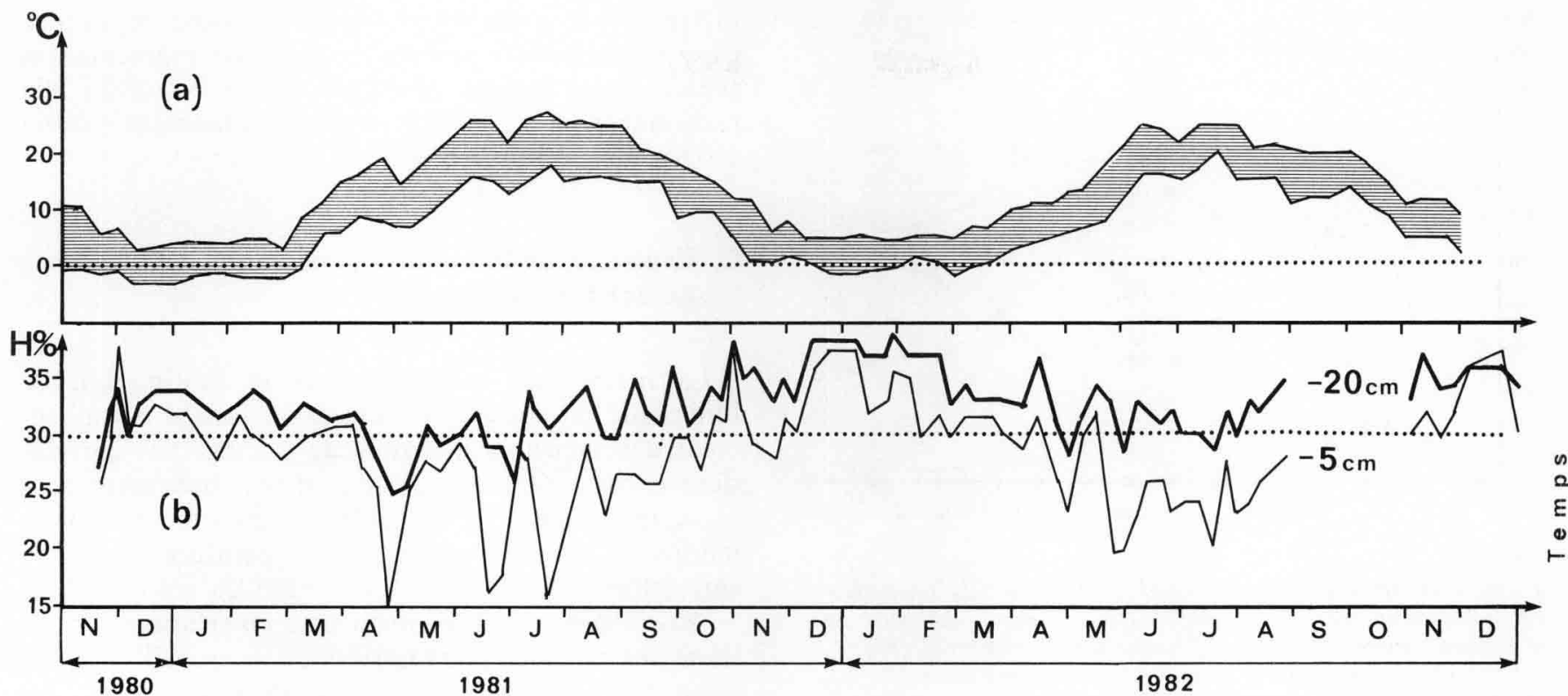

Figure 1

Température et humidité du sol: enregistrements effectués durant la période d'enfouissement des semences.

(a) Amplitude thermique (minimum et maximum enregistrés par décade) du sol à $-10 \mathrm{~cm}$.

(b) Humidité (exprimée en g d'eau pour $100 \mathrm{~g}$ de sol sec). Mesures hebdomadaires à -5 et $-20 \mathrm{~cm}$. La capacité de rétention de ce sol argilo-limoneux à $p F 2,8$ est voisine de $30 \%$; le point de flétrissement permanent ( $p F 4,2$ ) correspond à une humidité de $18 \%$.

\section{Humidité}

L'humidité du sol a été appréciée à -5 et $-20 \mathrm{~cm}$ durant toute la période expérimentale, par prélèvements hebdomadaires et pesée de la quantité d'eau par $100 \mathrm{~g}$ de terre sèche. Nous avons établi des moyennes sur $14,21,28$ et $42 \mathrm{j}$ qui nous ont permis, comme pour la température, de calculer les coefficients de corrélation entre la capacité germinative des semences et l'humidité du sol.

Dans tout ce qui suivra, nous réserverons le terme de "germinations " pour ce qui concerne la capacité germinative des semences estimées in vitro et le terme "levées" pour les germinations suivies de levées de plantules en plein champ.

\section{RÉSULTATS}

\section{A. Conservation des semences dans le sol (fig. 2)}

Aethusa et Euphorbia se conservent très bien dans le sol. Le taux de conservation des semences de vulpin diminue significativement entre 0 et $160 \mathrm{j}$ (85 p. 100 de semences morphologiquement normales) puis reste stable durant le reste de la période de conservation. La destruction des semences de matricaire est plus importante, elle est régulière et représente un taux de disparition annuel de 17 p. 100 . Chez ces 2 dernières espèces, l'embryon est susceptible de germer très rapidement (LONCHAMP \& GORA, 1980a), de croître puis d'être détruit entre 2 observations.

Temperature and soil moisture: data obtained throughout the burial period of the seeds.

(a) Range of soil temperature (minimum and maximum measured at $10 \mathrm{~cm}$ depth in soil over ten days).

(b) Soil moisture ( $g$ of water per $100 \mathrm{~g}$ of dry soil). Weekly measures at 5 and $20 \mathrm{~cm}$ depth. The retention ability of this clay silty soil at $p F=2.8$ is about $30 \%$; the welting point $(p F=4.2)$ corresponds to $18 \%$. 


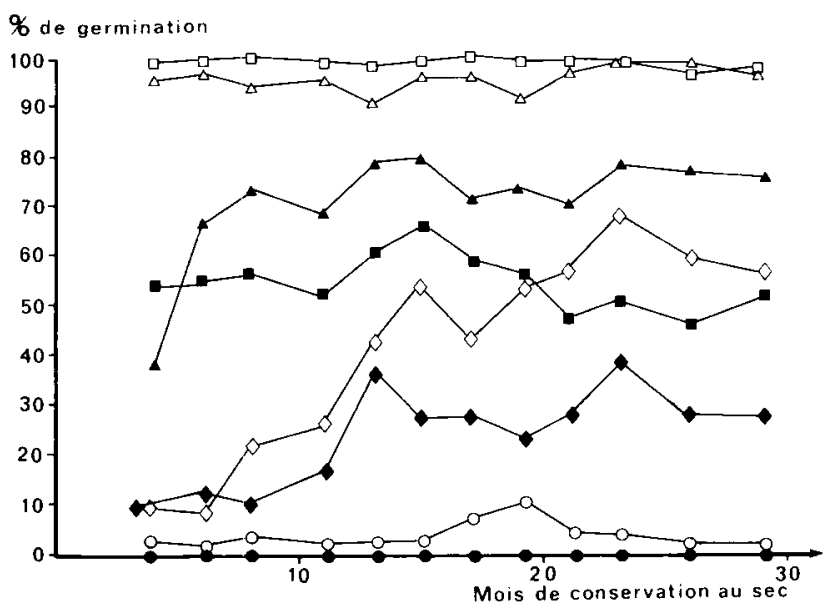

Figure 3

Capacité germinative à l'alternance $15-10^{\circ} \mathrm{C}$, à l'obscurité (figurés noirs) et en présence de lumière (figurés clairs), des semences $d$ 'Aethusa cynapium $(\bullet, \bigcirc)$, d'Alopecurus myosuroides $(\boldsymbol{\Lambda}, \Delta)$, $d$ 'Euphorbia exigua $(\bullet, \diamond)$ et de Matricaria perforata $(\boldsymbol{\square}, \square)$ après leur conservation au sec au laboratoire.

Germinative ability at $15-10{ }^{\circ} \mathrm{C}$ alternating temperature, in the dark (black points) and with light (white points) for Aethusa cynapium $(\bullet, \bigcirc)$, Alopecurus myosuroides $(\boldsymbol{\Delta}, \triangle)$, Euphorbia exigua $(\diamond, \diamond)$ and Matricaria perforata $(\boldsymbol{\square}, \square)$ seeds, after dry storage in the laboratory.

présence de lumière durant les 28 mois de la période de conservation au laboratoire. Les semences d'Euphorbia germent peu $(8$ p. 100) 3 mois après leur récolte, puis le taux de germination croît, pour atteindre des valeurs de 20 à 40 p. 100 à l'obscurité et 60 p. 100 à la lumière.

Les semences d'Alopecurus et de Matricaria, nettement photosensibles, germent à plus de $90 \mathrm{p}$. 100 en présence de lumière alors qu'à l'obscurité le taux de germination fluctue entre 40 et 65 p. 100 pour la matricaire et entre 60 et 80 p. 100 pour le vulpin. Chez cette dernière espèce, on note une augmentation rapide de la faculté germinative à l'obscurité ; elle reste stable par la suite et n'atteint jamais les valeurs observées à la lumière.

\section{Evolution de la capacité germinative des semences durant l'enfouissement}

La mesure de la température et l'estimation de l'humidité du sol à proximité de la zone d'enfouissement des semences a permis de calculer des corrélations entre différentes variables d'environnement et la capacité germinative. Le tableau 1 présente ces corrélations. Parmi ces valeurs, la température maximale enregistrée à $-10 \mathrm{~cm}$ et l'humidité du sol estimée à $-20 \mathrm{~cm}$ présentent les meilleures corrélations ; nous les avons retenues pour la suite de ce travail.

La durée de prise en compte de ces variables au moment de l'extraction et de l'établissement des tests de germination est importante au niveau du calcul des coefficients de corrélation (fig. $4 a$ et $b$ ).

Pour la température (fig. 4a), les coefficients de corrélation évoluent assez peu pour toutes les espèces et pour les conditions de test utilisées (à l'obscurité ou en présence de lumière), sauf pour le vulpin. Chez cette espèce, les coefficients croissent régulièrement de 0,340 à 0,580 lorsque l'on passe de 10 à $60 \mathrm{j}$ d'intégration.

Pour l'humidité du sol (fig. $4 b$ ), l'évolution des coefficients de corrélation est très importante entre les $7^{\mathrm{e}}$ et $21^{\mathrm{e}} \mathrm{j}$, moins par la suite.

A partir de ces observations, la durée d'intégration des variables de température et d'humidité a été fixée arbitrairement à $20 \mathrm{j}$ ( 21 pour l'humidité), quelles que soient les espèces étudiées.

\section{TABLEAU 1}

Valeur des coefficients de corrélation calculés entre la capacité germinative des différentes espèces en présence (Lum.) ou en absence (Obsc.) de lumière en 1981-82, et quelques variables d'environnement dont les movennes ont été calculées sur les $20 j$ qui ont précédé l'extraction. les * indiguent l'intensite de la liaison tsignifications à 5 , l et $0.1 \% \mathrm{~m})$.

Correlation coefficients between germinative ability and some environmental variables, calculated for a period of 20 days before the extraction of seeds. Germinative ability is measured under light (Lum.) or dark (Obsc.) conditions.

* indicate a significant value $(5, I$ and $0.1 \%)$.

\begin{tabular}{|c|c|c|c|c|c|c|c|c|}
\hline $\begin{array}{l}\text { Capacité } \\
\text { germinative } \\
\text { (1) }\end{array}$ & $\begin{array}{l}\text { Variables d'envi- } \\
\text { ronnement }\end{array}$ & $\begin{array}{c}\text { Durée } \\
\text { d'enfouis- } \\
\text { sement }\end{array}$ & $\begin{array}{l}\text { Minima } \\
-10 \mathrm{~cm}\end{array}$ & $\begin{array}{l}\text { Maxima } \\
-10 \mathrm{~cm}\end{array}$ & $\begin{array}{l}\text { Médiane } \\
-10 \mathrm{~cm}\end{array}$ & $\begin{array}{l}\text { Médiane } \\
\text { Abri }\end{array}$ & $\begin{array}{l}\mathrm{H} \% \\
-\quad 5 \mathrm{~cm}\end{array}$ & $\begin{array}{c}\mathrm{H} \% \\
-20 \mathrm{~cm}\end{array}$ \\
\hline Matricaria & Cibsc. & $0,815^{* * *}$ & $0,348^{*}$ & 0,294 & 0,319 & $0,380^{*}$ & 0,161 & $0,522 * * *$ \\
\hline Aethusa & Obsc. & $0,415^{*}$ & $-0,386^{*}$ & $-0,439^{* *}$ & $-0,411^{*}$ & $-0,377^{*}$ & $0,607^{* * *}$ & $0,651^{* * *}$ \\
\hline Euphorbia & Obsc. & 0,273 & $-0,567^{* * *}$ & $-0,588^{* * *}$ & $-0,580^{* * *}$ & $-0,524^{* * *}$ & $0,496^{* *}$ & $0,474^{* *}$ \\
\hline Euphorbia & Lum. & $0,542^{* * *}$ & $-0,456^{* *}$ & $-0,492^{* *}$ & $-0,472^{* *}$ & $-0,422^{* *}$ & $0,576^{* * *}$ & $0,604^{* * *}$ \\
\hline Alopecurus & Obsc. & $-0,036$ & 0,186 & 0,197 & 0,190 & 0,172 & 0,090 & 0,210 \\
\hline Alopecurus & Lum. & $-0,226$ & $0,416^{*}$ & $0,422^{* *}$ & $0,418^{* *}$ & $0,376^{*}$ & $-0,177$ & $-0,023$ \\
\hline
\end{tabular}

(1) Moyennes des capacités germinatives à 10 et $25 \mathrm{~cm}$ de profondeur sauf pour Euphorbia où la profondeur de $10 \mathrm{~cm}$ a été retenue. (1) Means of germinative abilities at 10 and $25 \mathrm{~cm}$ depth ; except for Euphorbia where the $10 \mathrm{~cm}$ value is used. 
(A) Température

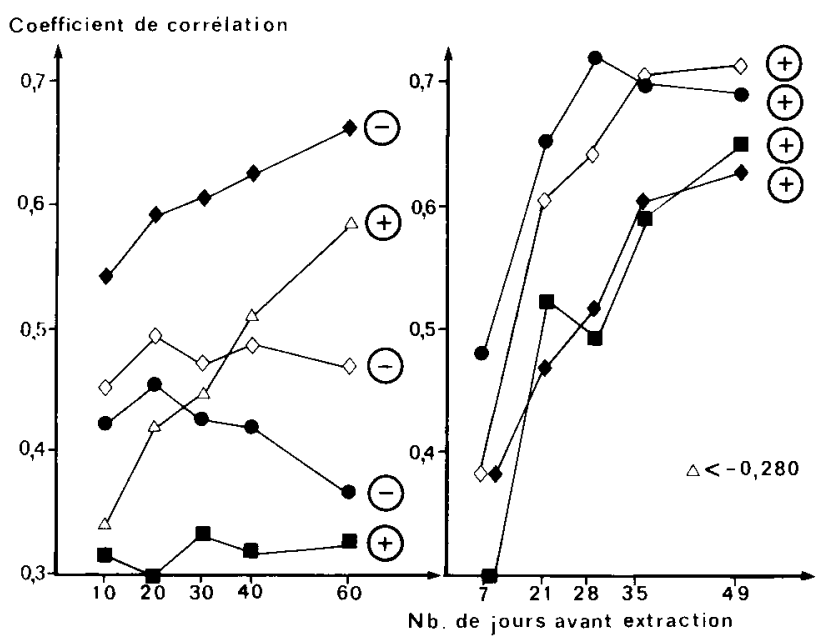

Figure 4

Evolution des coefficients de corrélation calculés entre la capacité germinative et les moyennes des températures maximales du sol à - $10 \mathrm{~cm}(A)$ ou les moyennes de l'humidité du sol à - $20 \mathrm{~cm}(B)$, estimées sur différentes périodes précédant l'extraction des semences.

- = Aethusa cynapium (Obsc.), $\Lambda=$ Alopecurus myosuroides $($ Lum. $), \diamond \diamond=$ Euphorbia exigua (Lum. et Obsc. $), \mathbf{\square}=$ Matricaria perforata (1.um.).

$\oplus$ et $\Theta=$ signe de la corrélation.

Correlation coefficients between the germinative ability and :

(A) the maximum soil temperatures means at $10 \mathrm{~cm}$ depth.

(B) the soil moisture means at $20 \mathrm{~cm}$ depth.

Means calculated for several periods before extraction of the seeds.

- = Aethusa cynapium (Darkness), $\triangle=$ Alopecurus myosuroides (Light), $\diamond \diamond=$ Euphorbia exigua (Light and Darkness), $\mathbf{\square}=$ Matricaria perforata (Light).

$\oplus$ and $\Theta=$ sign of correlation.

Le choix de la profondeur $20 \mathrm{~cm}$ pour l'humidité du sol par rapport à $5 \mathrm{~cm}$ se justifie parce que, d'une part, les valeurs des coefficients de corrélation sont souvent à l'avantage de cette mesure et, d'autre part, cette variable est peu corrélée aux variables de température (tabl. 2). Cette remarque nous permet d'utiliser dans des conditions satisfaisantes les calculs de régression multiple associant température et humidité du sol.

TABLEAU 2

Valeur des coefficients de corrélation entre les moyennes sur $20 ;$ des variables d'humidité et de température.

Les * indiquent l'intensité de la liaison (significations à 5,1 et $0 \%$ ).

Values of the correlation coefficients between soil moisture and soil temperature. The values averaged for a period of 20 days before the extraction of seeds.

* indicate a significant value $(5,1$ and $0.1 \%)$.

\begin{tabular}{|c|c|c|c|}
\hline $\begin{array}{c}\text { Tempé- } \\
\text { rature minima } \\
\text { Humidité }\end{array}$ & $\begin{array}{l}\operatorname{maxima} \\
-10 \mathrm{~cm}\end{array}$ & $\begin{array}{l}\text { médiane } \\
-10 \mathrm{~cm}\end{array}$ & $\begin{array}{l}\text { médiane } \\
\text { Abri }\end{array}$ \\
\hline $\mathrm{H} \% \dot{\mathrm{a}}-5 \mathrm{~cm}-0,656^{* * *}$ & $-0,682^{* * *}$ & $-0,671^{* * *}$ & $-0,580^{* * *}$ \\
\hline $\mathrm{H} \%_{0}$ à $-20 \mathrm{~cm}-0,322$ & $-0,374^{*}$ & $-0,351^{*}$ & $-0,259$ \\
\hline
\end{tabular}

\section{Matricaria perforata (fig. 5a)}

La capacité germinative des semences, estimée en présence de lumière dépasse 90 p. 100 durant les 2 années d'expérimentation.

A l'obscurité, la capacité germinative passe de 10 à 90 p. 100 en un an, puis reste stable. Cette augmentation du pouvoir germinatif est liée essentiellement à la durée de conservation (tabl. 1). Pour la seule année 1981 , le coefficient de corrélation atteint $0,932^{* * *}$.

\section{Aethusa cynapium (fig. $5 b$ )}

En présence de lumière, quelle que soit la profondeur d'enfouissement, la capacité de germination, voisine de 25 p. 100, reste stable jusqu'en avril 1981, diminue sensiblement en mai-juin, puis croît de juin à septembre pour rester constante et maximale jusqu'à la fin de l'expérience.

Pour l'année 1981, les corrélations avec la température $\left(r=0,587^{*}\right)$ et l'humidité $(r=0,226)$ ne sont pas suffisantes pour attribuer la perte puis la reprise de la germination à ces facteurs. Néanmoins, l'effet de l'humidité du sol sur l'induction de dormance sera abordé dans la discussion.

A l'obscurité, les tests de germination font apparaître une fluctuation saisonnière de la faculté germinative. En $1^{\text {re }}$ approximation, les semences sont capables de germer uniquement en période hivernale, la capacité de germination ainsi que la durée pendant laquelle la germination est possible augmentent avec le temps. En effet, en 1980-81, la germination est très faible mais significativement différente de 0 ; elle survient de janvier à mars. En 1981-82, elle se situe entre novembre et avril (maximum : 66 p. 100) ; en 1982-83 elle commence en octobre et atteint 70 p. 100 dès décembre.

Dans ces conditions de test, les taux de germination sont toujours à l'avantage des semences initialement enfouies à $10 \mathrm{~cm}$, ce qui suggère une influence marquée des facteurs d'environnement générateurs de gradients, telles la température et l'humidité. L'étude des corrélations (tabl. 1) fait apparaître une très bonne liaison avec l'humidité du sol et des liaisons moins importantes avec la température et la durée d'enfouissement. Il n'a pas été possible par l'étude des régressions multiples de dégager plus finement la participation de ces 3 facteurs.

\section{Euphorbia exigua (fig. 5c)}

L'évolution de la faculté germinative en présence ou en absence de lumière est semblable à ce qui a été décrit chez Aethusa à propos des tests réalisés à l'obscurité. Néanmoins, la profondeur d'enfouissement des semences ne joue un rôle qu'à l'obscurité.

Les semences sont capables de germer durant les mois d'hiver, mieux à l'obscurité qu'à la lumière au cours des 2 premières années, mais, au moment ou s'arrêtent nos observations, la photosensibilité semble

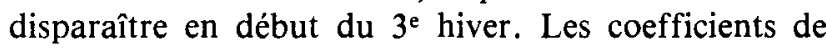
corrélation avec la température, calculés pour 198182 , sont meilleurs lors des tests réalisés à l'obscurité ; par contre en présence de lumière, les corrélations sont meilleures avec l'humidité (tabl. 1 et 3 ). Ces 

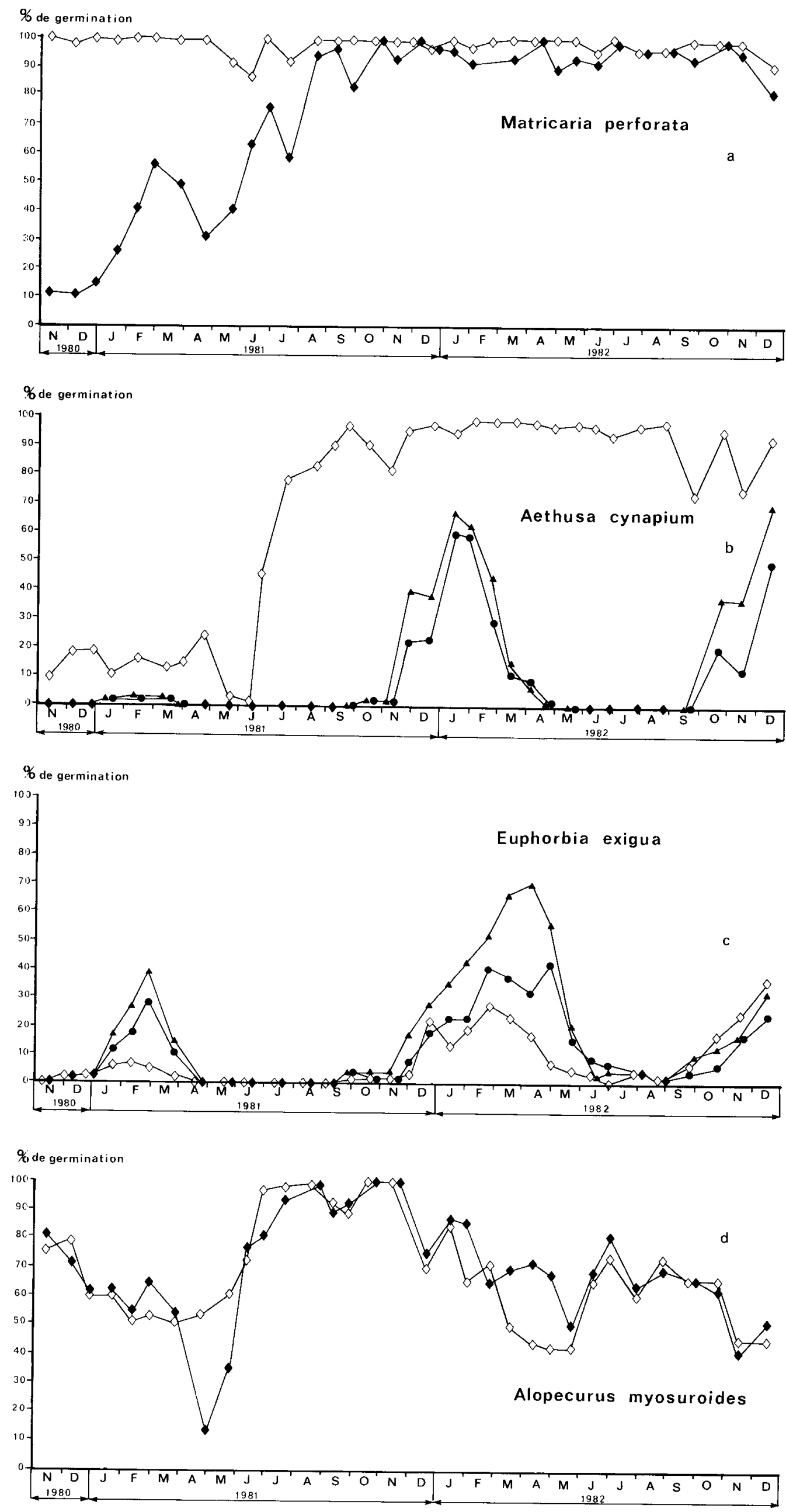
mêmes coefficients, calculés séparément pour 1981 et 1982 sont toujours favorables à une liaison avec la température (tabl. 3). Sur la période 1981-82, les corrélations multiples permettent d'intégrer la durée d'enfouissement, principalement lors des tests réalisés à la lumière (tabl. 4).

\section{Alopecurus myosuroides (fig. 5d)}

Contrairement à ceux des 2 espèces précédentes, les maxima de germination s'observent de juin à octobrenovembre. Les fluctuations de la faculté germinative ne manifestent une corrélation qu'en présence de lumière et exclusivement avec la température (fig. 1). Cependant, l'étude des régressions multiples permet d'intégrer l'effet de la durée d'enfouissement et de l'humidité (tabl. 4).

Les tests réalisés à l'obscurité mettent en évidence une chute de la capacité germinative en avril-mai 1981 que nous interpréterons dans la discussion.
TABLEAU 3

Valeur des coefficients de corrélation calculés pour 1981, 1982, $1981+1982$ entre la capacité de germination d'Euphorbia exigua, la température (Maxi à $-10 \mathrm{~cm})$, l'humidité $(\grave{a}-20 \mathrm{~cm})$ et la durée d'enfouissement (NJE).

Les * indiquent l'intensité de la liaison (significations a 5,1 et $0,1 \%$ ).

Value of the correlation coefficient between the germinative ability of Euphorbia exigua and soil temperature $(-10 \mathrm{~cm}$ Maxi), soil moisture $(-20 \mathrm{~cm})$ and length of burial (NJE).

* indicate a significant value $(5,1$ and $0.1 \%)$.

\begin{tabular}{|c|c|c|c|c|}
\hline & & 1981 & 1982 & $1981+82$ \\
\hline \multirow{3}{*}{ Obsc. } & Temp. & $-0,623^{* *}$ & $-0,732^{* * *}$ & $-0,588^{* * *}$ \\
\hline & $\mathrm{H} \%$ & 0,320 & 0,250 & $0,473^{* *}$ \\
\hline & NJE & $-0,342$ & $-0,458$ & 0,273 \\
\hline \multirow{3}{*}{ Lum. } & Temp. & $-0,758^{* * *}$ & $-0,704^{* *}$ & $-0,492^{* *}$ \\
\hline & H \% & 0,422 & 0,475 & $0,604^{* * *}$ \\
\hline & NJE & $-0,361$ & 0,045 & $0,541^{* * *}$ \\
\hline
\end{tabular}

TABLEAU 4

Equations de régressions linéaires simples ou multiples entre la faculté germinative des semences et 3 facteurs explicatifs : la durée d'enfouissement (NJE), l'humidité du sol à - $20 \mathrm{~cm}(H \%)$ et la moyenne des maxima à - $10 \mathrm{~cm}$ (Temp.).

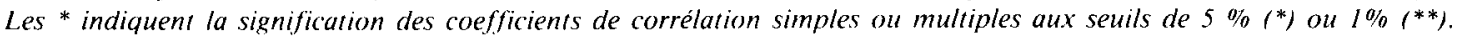
Simple or multiple linear regressions between germinative ability of seeds and length of burial (NJE), soil moisture at $20 \mathrm{~cm}$ depth (H\%) and soil temperature at $10 \mathrm{~cm}$ depth (Temp.).

$*$ indicate a significant value of the simple or multiple correlation coefficients $(5 \%: * ; 1 \%: * *)$.

\begin{tabular}{|c|c|c|c|c|c|c|c|c|}
\hline \multirow{2}{*}{\multicolumn{2}{|c|}{ Capacité germinative $\left({ }^{1}\right)$}} & \multicolumn{2}{|c|}{$1^{\text {er }}$ facteur } & \multicolumn{2}{|c|}{$2^{e}$ facteur } & \multicolumn{2}{|c|}{$3^{\text {e }}$ facteur } & \multirow{2}{*}{ Droites de régression } \\
\hline & & Nature & Corrél. & Nature & Corrél. & Nature & Corrél. & \\
\hline Matricaria & Obsc. & NJE & $0,815^{* *}$ & & & & & $\mathrm{y}=31,7+0,1 \mathrm{x}_{\mathrm{NJE}}$ \\
\hline Aethusa & Obsc. & $\mathrm{H} \%$ & $0,651^{* *}$ & & & & & $\mathrm{y}=-150,6+4,8 \mathrm{x}_{\mathrm{H} \%}$ \\
\hline Euphorbia & Obsc. & Temp. & $0,588^{* *}$ & NJE & $0,736^{* *}$ & & & $\begin{aligned} \mathrm{y}=24,5 & -1,9 \mathrm{x}_{\mathrm{Temp}} . \\
& +0,04 \mathrm{x}_{\mathrm{NJE}}\end{aligned}$ \\
\hline Euphorbia & Lum. & $\mathrm{H} \%$ & $0,604^{* *}$ & NJE & $0,673^{* *}$ & & & $\begin{array}{c}y=-58,5+1,8 x_{H \%} \% \\
+0,02 x_{\text {NJE }}\end{array}$ \\
\hline Alopecurus & Lum. & Temp. & $0,422^{*}$ & NJE & $0,547^{* *}$ & $\mathrm{H} \%$ & $0,718^{* *}$ & $\begin{aligned} y & =-87,8+2,0 \times \text { XTemp } \\
& -0,06 x_{\text {NJE }}+4,7 x_{H}\end{aligned}$ \\
\hline
\end{tabular}

(1) Moyennes des capacités germinatives à 10 et $25 \mathrm{~cm}$ de profondeur, sauf pour Euphorbia où la profondeur de $10 \mathrm{~cm}$ a été retenue. (1) Means of germinative abilities at 10 and $25 \mathrm{~cm}$ depth ; except for Euphorbia where the $10 \mathrm{~cm}$ value is used.

\section{Figure 5}

Evolution de la capacité germinative des semences de Matricaria perforata (a), d'Aethusa cynapium (b), $d^{\prime}$ Euphorbia exigua (c) et $d$ 'Alopecurus myosuroides (d) au cours de leur conservation dans le sol. Les semences ont été préalablement placées à 10 (4) et 25 (•) $\mathrm{cm}$ de profondeur; dans le cas où aucune différence de la capacité germinative n'apparaît entre ces profondeurs on a représenté leur moyenne $(\diamond, \diamond)$. Tests à $15-10^{\circ} \mathrm{C}$, à l'obscurité (figurés noirs) et en présence de lumière (figurés clairs).

Date d'enfouissement : 20-10-1980.

Germinative ability of Matricaria perforata (a), Aethusa cynapium (b). Euphorbia exigua (c) and Alopecurus myosuroides (d) seeds, during the entire period of burial. The depths of burial were 10 (A) and $25 \mathrm{~cm}(\bullet)$. When no difference between the depths were found, means are presented $(\diamond, \diamond)$. Tests at $15-10^{\circ} \mathrm{C}$, in the dark (black points) and with light (white points).

Burial date : 20-10-1980.

\section{Chronologie des levées au champ (fig. 6 et 7 )}

\section{Matricaria perforata}

En peuplement artificiel (monocultures), les levées apparaissent dès mi-avril jusqu'à la fin juin avec un maximum en avril-mai au cours des 3 années d'observation. Cette espèce est absente du peuplement naturel (cultures assolées).

\section{Aethusa cynapium}

En peuplement artificiel, comme en peuplement naturel, les levées débutent dès mars pour se terminer 

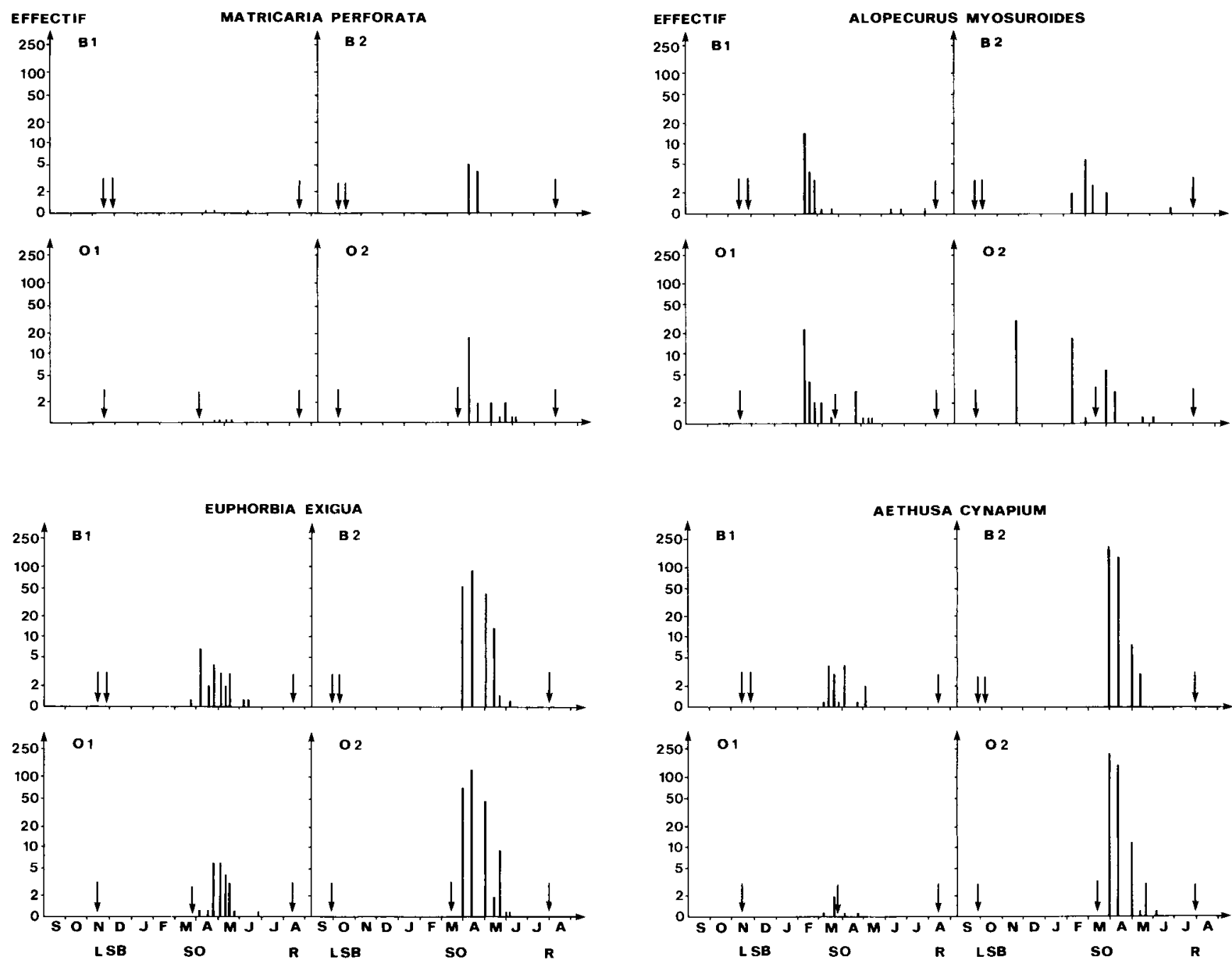

Figure 6

Chronologie des levées (exprimées en nombre d'individus $/ \mathrm{m}^{2}$ ) dans un peuplement artificiel sous monocultures de blé d'hiver et d'orge de printemps, au cours des 2 années culturales 1979-80 et 1980-81.

$B_{1}=$ sous blé d'hiver en 1979-80

$B_{2}=$ sous blé d'hiver en 1980-81

$O_{1}=$ sous orge de printemps en 1979-80

$\mathrm{O}_{2}=$ sous orge de printemps en 1980-81

Les dates de labour, de semis de blé et d'orge, de récolte sont représentées respectivement par $L, S B$, SO et $R$.

fin avril durant les 2 premières années d'observation. En $3^{\mathrm{e}}$ année, les levées ont été plus précoces en peuplement artificiel.

\section{Euphorbia exigua}

En peuplement artificiel, l'époque des levées est assez étalée : de mi-mars à fin juin pour les 2 premières années. En $3^{\mathrm{e}}$ année, on observe des levées tardives (début août).

En peuplement naturel, les levées ne sont plus groupées, probablement en raison de la faible infestation de cette parcelle pour laquelle le nombre d'observations n'est pas suffisant.

\section{Alopecurus myosuroides}

En peuplement artificiel, après le labour et avant le semis de l'orge, on observe des levées dès la fin de

Emergence dates (expressed as individuals per $\mathrm{m}^{2}$ ) of sown seeds under winter wheat and spring barley monocultures, during two years of cropping.

$B_{1}=$ winter wheat in 1979-80

$B_{2}=$ winter wheat in 1980-81

$O_{1}=$ spring barley in 1979-80

$\mathrm{O}_{2}=$ spring barley in 1980-81

The dates of ploughing, wheat and barley sowing, harvest are marked as $L, S B, S O, R$, respectively.

l'été avec un maximum en automne. L'absence de ces levées sous monoculture de blé durant cette période est probablement due aux façons culturales préparatoires au semis qui auraient détruit les plantules en cours de levée.

En peuplement naturel, les quelques levées inventoriées (en raison du faible potentiel en semences du sol) apparaissent au printemps. L'absence d'observations en inter-culture ne permet pas les comparaisons entre les 2 peuplements.

\section{DISCUSSION}

\section{A. Méthodologie}

Les variables utilisées pour le calcul des corrélations avec la capacité germinative ont été mesurées ou estimées à des profondeurs généralement différentes de 


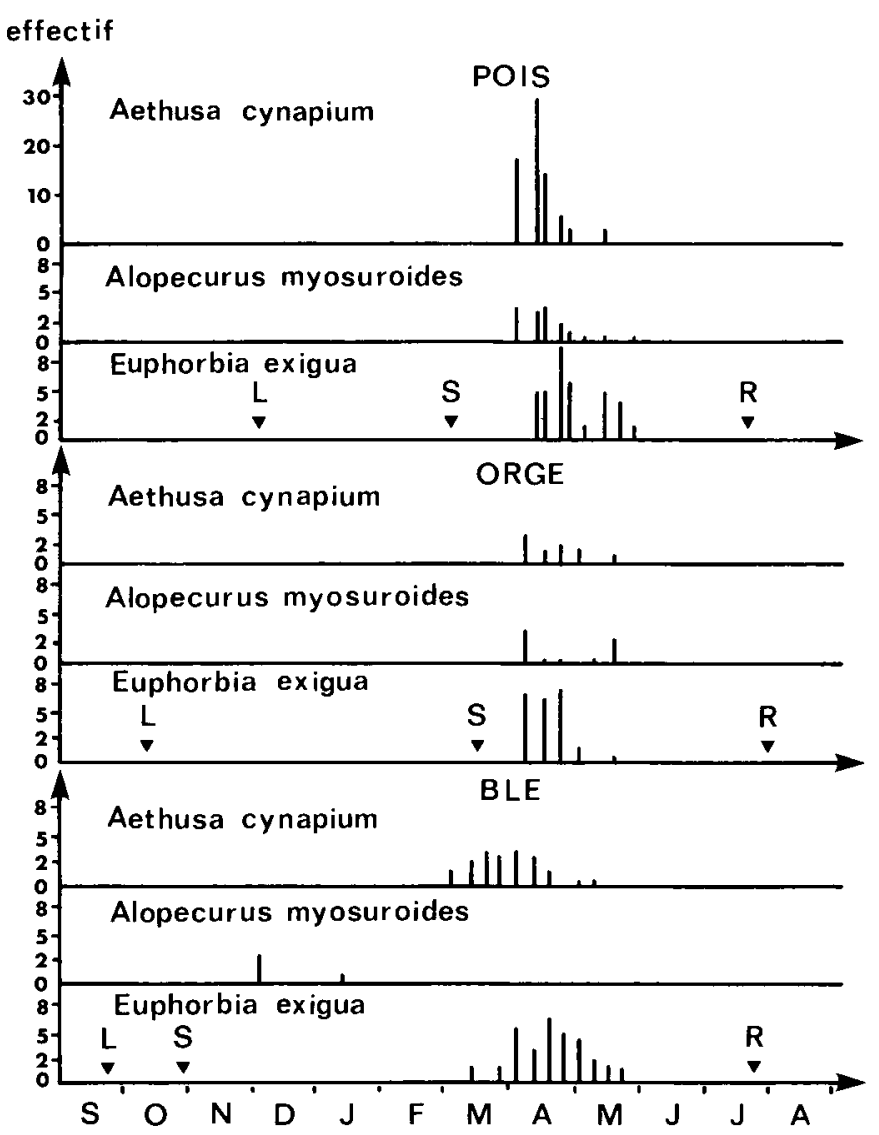

Figure 7

Chronologie des levées (exprimées en nombre d'individus $/ \mathrm{m}^{2}$ ) dans un peuplement naturel au cours des années culturales 1979-80 (blé d'hiver), 1980-81 (orge de printemps), 1981-82 (pois de printemps). Les dates de labour, de semis et de récolte sont représentées respectivement par $L, S$ et $R$.

Emergence dates (expressed as individuals per $\mathrm{m}^{2}$ ) of natural weed seeds during three years of cropping : 1979-80 (winter wheat), 198081 (spring barley), 1981-82 (peas).

The dates of ploughing, sowing and harvest are marked as $L, S$ and $R$ respectively.

celles où les semences ont été conservées. Cette différence dans les niveaux d'observation s'est avérée sans incidence. En effet, pour le vulpin et la matricaire (à la lumière et à l'obscurité), la petite ciguë et l'euphorbe (à la lumière), il n'y a pas de différence significative de germination entre des semences préalablement enfouies à 10 ou $25 \mathrm{~cm}$. Dans les cas où une différence a été observée (petite ciguë et euphorbe à l'obscurité), les coefficients de corrélation sont très proches. Ces constatations nous amènent à réfléchir sur la finesse des relations entre certains facteurs de l'environnement et les phénomènes de dormance observés chez les semences.

L'humidité du sol, telle que nous l'avons estimée (contenu en eau), ne reflète pas les effets directs de l'eau au niveau des semences (imbibition, vecteur de métabolites solubles, volatils ou non). De plus, la technique des prélèvements hebdomadaires ne renseigne pas sur les fluctuations effectives du facteur.

A propos de la durée pendant laquelle les variables d'un facteur doivent être prises en compte avant extraction des semences, la figure 4 est déconcertante. La durée d'intégration (excepté l'humidité sur 7 j) influence assez peu la valeur des coefficients de corré- lation et l'on peut se demander ce que représente biologiquement une moyenne de températures établie sur $60 \mathrm{j}$. Ces études de corrélations devront être approfondies de façon à affiner les liaisons déjà évoquées et préciser le synchronisme des variables et de l'état des semences.

Les corrélations humidité-température du substrat de conservation sont souvent importantes (tabl. 2) et les régressions multiples calculées par la méthodé progressive (BACHACOU et al., 1981) sont peu adaptées pour expliquer l'évolution de la capacité germinative. Les droites de régression ont été calculées (tabl. 4) ; elles renseignent peu sur la part de l'un ou l'autre des facteurs dans l'évolution de la capacité germinative, mais elles permettent d'estimer assez grossièrement le comportement des semences.

Une analyse en composantes principales a été effectuée sur les valeurs prises par 3 variables dans les $20 \mathrm{j}$ qui précèdent les extractions : moyennes des maxi à $-10 \mathrm{~cm}$, humidité à $-20 \mathrm{~cm}$ et ETP (l'évapotranspiration potentielle calculée suivant la formule de PENMAN, estime la capacité évaporante de l'air). Les composantes 1 et 2 absorbent 97 p. 100 de la variabilité totale (tabl. 5) et la représentation des dates d'extraction dans ce plan permet de caractériser climatiquement celles-ci (fig. 8). On constate alors que la chute de la capacité germinative du vulpin et de la matricaire à l'obscurité, de la petite ciguë à la lumière pour les extractions d'avril-juin 1981 correspond à un printemps chaud et sec dont nous discuterons l'incidence sur l'induction de dormances.

TABLEAU 5

Comparaison des composantes principales. Comparison of principal components.

\begin{tabular}{ccccc}
\hline \hline & & \multicolumn{3}{c}{ Composantes principales } \\
\cline { 2 - 5 } & & 1 & 2 & 3 \\
\hline$\%$ d'inertie absorbé par les axes & 72,7 & 24,3 & 3,0 \\
\hline $\begin{array}{c}\text { Coefficients } \\
\text { de } \\
\text { corrélation }\end{array}$ & Maxima -10 & 0,932 & 0,296 & $-0,208$ \\
\cline { 2 - 5 } & H $\%-20$ & $-0,641$ & 0,767 & 0,016 \\
\cline { 2 - 5 } & ETP & 0,950 & 0,228 & 0,216 \\
\hline \hline
\end{tabular}

\section{B. Evolution de la capacité germinative}

D’une façon générale, les variations de la capacité germinative des semences sont assez lentes et nous avons déjà constaté que la durée d'intégration des facteurs explicatifs pouvait varier, pour la température par exemple, de 10 à $60 \mathrm{j}$ sans grande incidence sur les coefficients de corrélation.

La faculté germinative peut être d'emblée maximale (matricaire à la lumière), ou croître régulièrement (matricaire à l'obscurité et petite ciguë à la lumière). Dans ce cas, l'évolution peut être modulée par des accidents climatiques (sécheresse d'avril-mai 1981).

L'évolution de la capacité de germination peut être continue ou cyclique suivant les espèces et les modalités de mise en germination (HORNG \& LEU, 1978 ; 


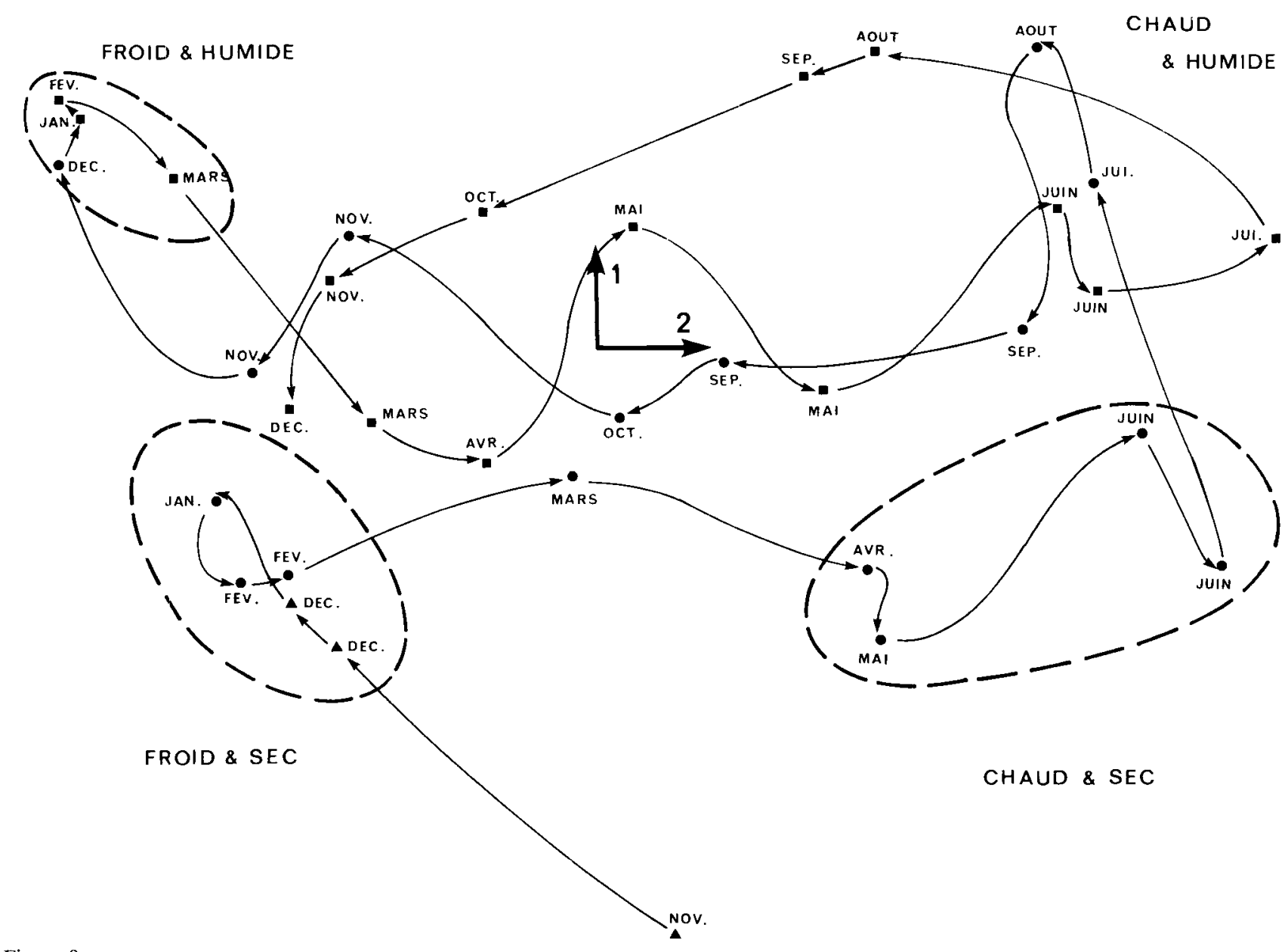

Figure 8

Représentation des dates d'extraction suivant les composantes principales 1 et 2.

Chadoeuf et al., 1980). Chez l'euphorbe et la petite ciguë (à l'obscurité), la capacité germinative manifeste une évolution cyclique de périodicité annuelle ; de telles fluctuations ont souvent été observées.

L'augmentation des possibilités de germination d'A. cynapium est essentiellement hivernale et rappelle le comportement des semences d'Euphorbia supina Rafin. et de Lamium amplexicaule L. (BASKIN \& BASKIN, 1979, 1981b). E. exigua est capable de germer de la fin de l'automne au début du printemps comme Polygonum aviculare L. (COURTNEY, 1968), Cyperus inflexus Muhl. et Verbascum blattaria L. (BASKIN \& BASKIN, 1978, 1981a). Pour ces 2 dernières espèces, l'époque de levée au champ correspond bien à la période maximale de germination obtenue en laboratoire (fig. $5 b$ et $c$ ) si l'on tient compte du décalage (estimé approximativement à 1 mois et demi) entre la date de l'extraction des semences pour l'étude de la germination et la date de l'apparition au champ des plantules dont la croissance de l'hypocotyle est lente.

L'évolution de la capacité germinative du vulpin en présence de lumière n'a pu être clairement définie, mais il semble qu'elle soit du type cyclique avec un maximum de germination en été-automne, comparable à ce qui est observé chez Aphanes arvensis L. (ROBERTS \& NEILSON, 1982).

La germination de la matricaire inodore peut
Sampling dates in the first two principal components. $\mathbf{\Delta}=1980 \quad \bullet=1981 \quad \mathbf{Q}=1982$

s'effectuer à l'obscurité dans de bonnes conditions à partir de l'été qui suit la récolte des semences, mais, au champ, les levées ne se manifestent que pendant la période mi-avril à fin-juin. En effet, d'une part les graines ont besoin de températures assez élevées pour germer (LONCHAMP \& GORA, 1980a), ce qui expliquerait l'apparition des premières levées seulement en avril, d'autre part, pendant la période avril-juin, toutes les semences placées dans des conditions d'environnement favorables ont germé, ce qui expliquerait l'absence de levées après cette période. ROBERTS \& NEILSON (1981) ont observé, sur des espèces voisines (Matricaria recutita $\mathrm{L}$. et $M$. discoidea DC.) des levées d'avril à octobre à la suite de brassages épisodiques du sol, ce qui permet de replacer des semences en conditions de germination, donc d'allonger la période des levées. Enfin, les températures élevées de l'été pourraient induire une dormance secondaire, comme l'a montré COURTNEY (1968) chez $P$. aviculare.

Généralement les semences présentes dans le sol se conservent bien; elles sont incapables de germer in situ (LONCHAMP \& GORA, 1980b). A cette inhibition de germination se superpose une dormance induite puis levée par les conditions d'environnement (RoBERTS, 1981 ; KARSSEN, 1982). Au champ, il est logique de penser que les semences germent :

1) lorsqu'un travail du sol les place près de la surface, 
2) si d'éventuelles dormances ont été levées,

3) si la température et l'humidité du sol sont compatibles avec leurs exigences germinatives.

A l'obscurité (situation la plus fréquente pour les adventices de cultures annuelles), les semences d'E. exigua et d'A. cynapium, espèces à levée printanière (MONTEGUT, 1975 ; ROBERTS, 1979), germent préférentiellement à basses températures (LONCHAMP \& GORA, 1980), donc à l'époque où leur capacité germinative est la plus élevée. Le vulpin, espèce à germination automnale préférentielle ou pré-printanière (Lovato \& VigGiani, 1974 ; MonTEGUT, 1975), germe dans une gamme de températures assez large dont l'optimum se situe, pour des semences sèches, vers $15-20^{\circ} \mathrm{C}$. Pour ces 3 espèces, la période de levée au champ s'explique donc bien par les exigences thermiques de levée de dormance et de germination.

Il n'y a cependant pas toujours correspondance entre les époques de levée de dormance et les possibilités thermiques de germination. Chez $V$. blattaria, BASKIN \& BASKIN (1981a) montrent que la capacité de germination est maximale en hiver-printemps et que les semences enfouies germent mieux à hautes qu'à basses températures. ROBERTS \& LOCKETT (1978), CHADOEUF et al. (1980) observent que les semences de Veronica hederifolia L. possèdent une capacité germinative élevée de septembre à décembre ; bien que pouvant germer à températures plus basses que celles qu'on relève d'habitude dans le sol à cette époque de l'année, l'enfouissement rend possible la germination de ces 2 espèces à des températures non optimales, favorisant un étalement des levées dans le temps.

\section{Effets de l'humidité du sol et de la température sur la capacité germinative}

LONCHAMP \& BARRALIS (1983) ont montré, sur semences sèches de $M$. perforata et $A$. myosuroides, que de faibles potentiels hydriques peuvent induire une dormance chez ces 2 espèces, dormance levée totalement chez la matricaire et partiellement chez le vulpin par l'action de la lumière. Sur semences préalablement enfouies, nous constatons qu'une dessiccation du sol en avril, juin et juillet (fig. 1) est corrélée à une baisse de la capacité germinative à l'obscurité chez la matricaire. Chez le vulpin, cette corrélation n'est décelable qu'en avril-mai. L'année 1982, très pluvieuse ne permet pas ces observations.

Chez les semences manifestant une fluctuation cyclique de leur pouvoir germinatif, la température de conservation dans le sol est à priori déterminante. COURTNEY (1968) induit une dormance chez $P$. aviculare par maintien des semences dans le sol à $25^{\circ} \mathrm{C}$. Cependant, chez $P$. aviculare comme chez $E$. exigua et $A$. cynapium, on observe une diminution de la capacité germinative dès mars-avril, mois pour lesquels la température du sol à $10 \mathrm{~cm}$ ne dépasse pas $20^{\circ} \mathrm{C}$. Il n'est donc pas certain que l'effet des hautes températures soit déterminant. Les basses températures par contre favorisent la levée de dormance chez l'euphorbe.

La différence de comportement germinatif des semences d'Aethusa à l'obscurité et à la lumière peut aussi être attribuée aux basses températures.
En effet, TAYLORSON \& HENDRICKS (1969), à la suite d'études sur Amaranthus retroflexus L., suggèrent 1) qu'à basses températures $\left(<20^{\circ} \mathrm{C}\right.$ pour l'amaranthe) il y a augmentation de la synthèse de phytochrome sous forme active ; 2) qu'à températures élevées ce même phytochrome subit une réversion. Il s'ensuit que les semences peuvent germer à l'obscurité après un conditionnement à basses températures et qu'à températures élevées elles ont besoin d'un apport de lumière.

Si la stratification (au sens des effets associés du froid et de l'enfouissement) se substitue partiellement au besoin de la lumière, son effet est particulier sur l'euphorbe puisqu'il y a inversion de la photosensibilité suivant que les semences sont conservées au sec (fig. 3) ou qu'elles sont enfouies (fig. 5c).

Enfin, l'évolution de la capacité germinative de cette dernière espèce illustre une apparente hétérogénéité de l'échantillon des semences utilisées : celles-ci germent plutôt en présence de lumière qu'à l'obscurité et ce décalage semble s'accentuer avec la durée de conservation dans le sol (fig. $5 c$ ). Ce même phénomène a été observé sur E. supina et $L$. amplexicaule (BASKIN \& BASKIN, 1979 et $1981 b$ ).

\section{CONCLUSION}

Nous avons essayé, comme de nombreux auteurs, de rapprocher les possibilités de germination des semences aux levées généralement observées dans des parcelles cultivées ou travaillées épisodiquement. Chez $A$. cynapium et $E$. exigua, les périodes de minimum de dormance correspondent effectivement aux époques de levées ; chez $M$. perforata et $A$. myosuroides les facteurs climatiques et culturaux expliquent l'absence de levées à certaines périodes.

L'environnement des semences conservées dans le sol conditionne leur comportement germinatif différemment suivant les espèces. Au laboratoire, il est possible de simuler l'action des facteurs de conservation et généralement on obtient assez rapidement (quelques jours) un effet significatif sur la capacité germinative des semences traitées.

Dans nos expériences d'enfouissement de semences, il a été possible d'établir des corrélations entre l'état dormant et la variation de certains facteurs d'environnement. Cependant, ces derniers étant généralement liés entre eux, il est difficile d'apprécier la participation de chacun. De plus, les effets de compensation (remplacement), de synergie entre facteurs compliquent l'interprétation. La mise en place au laboratoire de dispositifs factoriels simples devrait permettre d'affiner les interprétations des études de plein champ.

En effet, au champ, les façons culturales, labours, hersages, confèrent au sol structure et porosité en même temps qu'ils déplacent verticalement les semences. Les facteurs d'environnement n'agissent plus de façon continue et n'exercent pas une pression d'induction de dormance suffisante pour provoquer sur les semences enfouies une réaction rapide et totale, ce qui peut expliquer l'évolution assez lente de la capacité germinative et un étalement des levées dans le temps. 
Les populations de mauvaises herbes sont généralement représentées dans les parcelles agricoles par des semences d'âges différents et pour lesquelles la durée de l'enfouissement peut jouer un rôle important. Cependant, l'observation des levées de plantules issues de semences récemment introduites ou présentes depuis plusieurs années (stock semencier) montre un synchronisme qui suggère une réaction identique des semences aux facteurs d'environnement.

Reçu le 23 décembre 1983. Accepté le 26 mars 1984.

\section{RÉFÉRENCES BIBLIOGRAPHIQUES}

Bachacou J., Masson J.-P., Millier C., 1981. Manuel de la programmathèque statistique AMANCE 81. Dépt. de Biométrie, I.N.R.A., 516 p.

Baskin J.-M., Baskin C. C., 1978. Seasonal changes in the germination response of Cyperus inflexus seeds to temperature and their ecological significance. Bot. Gaz., 139, 231-235.

Baskin J.-M., Baskin C. C., 1979. Timing of seed germination in the weedy summer annual Euphorbia supina. Bartonia, 46, 63-68.

Baskin J.-M., Baskin C. C., 1981a. Seasonal changes in germination of buried seeds of Verbascum thapsus and V. blattaria and ecological implications. Can. J. Bot., 59, 1769-1775.

Baskin J.-M., Baskin C. C., 1981b. Seasonal changes in the germination responses of buried Lamium amplexicaule seeds. Weed Res., 21, 299-306.

Chadouf R., Magnière J.-P., Lonchamp J.-P., Barralis G., 1980. Evolution comparée de la faculté germinative de semences de mauvaises herbes enfouies ou conservées au sec. VIe Coll. Inter. Ecol. Biol. et Syst. des Mauvaises Herbes, Montpellier, 103-112.

Courtney R. D., 1968. Seed dormancy and field emergence in Polygonum aviculare. J. Appl. Ecol., 5, 675-683.

Horng L. C., Leu L. S., 1978. The effects of depth and duration of burial on the germination of ten annual weed seeds. Weed Sci., 26, 4-10.

Karssen C. M., 1982. Seasonal patterns dormancy in weed seeds, 243-270. In KHAN A. A. "The physiology and biochemistry of seed development, dormancy and germination. Elsevier Biomedical Press, $547 \mathrm{p}$.

Lovato A., Viggiani P., 1974. Germinabilità in laboratorio ed emergenza in campo di alcune specie infestanti. Riv. di Agron., 8, 108-112.

Lonchamp J.-P. Barralis G., 1983. Effets de faibles potentiels hydriques sur les possibilités de germination de semences d'Alopecurus myosuroides Huds. et de Matricaria perforata Mérat. Agronomie, 3, 435-441.

Lonchamp J.-P., Gora M., 1980a. Evolution de la faculté germinative de semences de mauvaises herbes au cours de leur conservation au sec. I.N.R.A., Lab. Malherbol., BV 1540, 21034 Dijon Cedex, $66 \mathrm{p}$.
Lonchamp J.-P., Gora M., 1980b. Effet de l'enfouissement sur les exigences germinatives de mauvaises herbes. VI ${ }^{e}$ Coll. Inter. Ecol. Biol. et Syst. des Mauvaises Herbes, Montpellier, 113-122.

Montegut J., 1975. Ecologie de la germination des mauvaises herbes, 192-217. In Chaussat R., Le DeunfF Y., La germination des semences. Gauthier-Villars, $232 \mathrm{p}$.

Popay A. I., Roberts E. H., 1970. Ecology of Capsella hursa pastoris $\mathrm{L}$. and Senecio vulgaris $\mathrm{L}$. in relation to germination behaviour. J. Ecol., 58, 123-138.

Roberts E. H., 1981. The interaction of environmental factors controlling loss of dormancy. Ann. Appl. Biol., 98, 552-555.

Roberts H. A., 1979. Periodicity of seedling emergence and reed survival in some Umbelliferae. J. Appl. Ecol., 16, 195-201.

Roberts H. A., Lockett P. M., 1978. Seed dormancy and periodicity of seedling emergence in Veronica hederifolia L., Weed Res., 18, $41-48$.

Roberts H. A., Neilson J. E., 1981. Seed survival and periodicity of seedling emergence in twelve weedy species of Compositae. Ann. Appl. Biol., 97, 325-334.

Roberts H. A., Neilson J. E., 1982. Seasonal changes in the temperature requirements for germination of buried seeds of Aphanes arvensis L., New Phytol., 92, 159-166.

Stoller E. W., Wax L. M., 1974. Dormancy changes and fate of some annual weed seeds in the soil. Weed Sci., 22, 151-155.

Taylorson R. B., 1970. Changes in dormancy and viability of weed seeds in soils. Weed Sci., 18, 265-269.

Taylorson R. B., 1972. Phytochrome-controlled changes in dormancy and germination of buried weed seeds. Weed Sci., 20, 417-422.

Taylorson R. B., Hendricks S. B., 1969. Action of phytochrome during prechilling of Amaranthus retroflexus L. seeds. Plant Physiol., 44, 821-825.

Wesson G., Wareing P. F., 1969. The role of light in the germination of naturally occurring populations of buried weed seeds. J. Exp. Bot., 20, 402-413. 